\title{
Global Longitudinal Dependence Observation of the Neutral Wind and Ionospheric Density Distribution
}

\author{
Endawoke Yizengaw \\ Institute for Scientific Research, Boston College, Chestnut Hill, MA, USA \\ Correspondence should be addressed to Endawoke Yizengaw, kassie@bc.edu \\ Received 4 November 2011; Revised 3 February 2012; Accepted 17 February 2012 \\ Academic Editor: Y. Sahai
}

Copyright ( $) 2012$ Endawoke Yizengaw. This is an open access article distributed under the Creative Commons Attribution License, which permits unrestricted use, distribution, and reproduction in any medium, provided the original work is properly cited.

\begin{abstract}
The statistical global view of the low-latitude ionospheric density stimulates further interest in studying the strong longitudinal variability of the ionospheric density structures in low-to-equatorial latitudes. However, we are not completely certain how the electrodynamics and ion-neutral coupling proceeds at low latitudes; in particular, the longitudinal difference in the dynamics of plasma structures in the low-to-mid latitude ionosphere is not yet fully understood. Numerical studies of latent heat release in the troposphere have indicated that the lower atmosphere can indeed introduce a longitudinal dependence and variability of the low-latitude ionosphere during quiet conditions. For the first time, we present simultaneous observations of the tidally modulated global wind structure, using TIDI observations, in the E-region and the ionospheric density distribution using ground (global GPS receivers) and space-based (C/NOFS in situ density and GPS TEC on CHAMP) instruments. Our results show that the longitudinally structured zonal wind component could be responsible for the formation of wave number four pattern of the equatorial anomaly.
\end{abstract}

\section{Introduction}

The plasma in the low-latitude ionosphere between $\pm 30^{\circ}$ magnetic latitude exists entirely on closed field lines and so is relatively cut off from magnetospheric and solar-wind drivers compared to mid- and high-latitude regions of the ionosphere. Most frequently, the highly populated plasma density in this region, which may be about two-thirds of all of the plasma in geospace, can become a disturbed space environment and disrupt the detection and tracking of aircraft, missiles, satellites, and other targets, distort communication and navigation, and interfere with global command, control, and surveillance operations. The plasma in the low and equatorial latitudes is disturbed or redistributed by transport mechanisms that form enhanced density located several degrees to either side of the magnetic equator, forming the equatorial ionization anomaly (EIA). The vertical drift (primarily $\mathbf{E} \times \mathbf{B}$ drift) is the primary plasma transport mechanism at low-to-equatorial latitudes. There are few cases reported on the night side $[1,2]$ as the EIA formation process is predominantly active on the dayside.
In the evening sector, when the conductivity becomes stronger at the terminator, the plasma redistribution is intensified again, forming a stronger EIA structure known as the prereversal enhancement. The redistribution of plasma occurs both during disturbed and quiet periods. During magnetically quiet periods, the tidal wind motions in the dayside E-region, which drive dayside Pedersen currents that produce charge build-up along the terminator, serve as the main source of dayside eastward electric field or vertical $\mathbf{E} \times$ B drift ([3] and the references therein). The question is, for the same local time sector, why does the plasma redistribution process behave differently from one longitudinal sector to the other? There is clear evidence that the formation of the global wave number-four structures, which is believed to be due to the different vertical $\mathbf{E} \times \mathbf{B}$ drift magnitude at different longitudes, in the longitudinal density distribution. Since Sagawa et al. [4] reported the formation of the four cells for the first time, different groups have continued to report the existence of the global wave number-four structures using observations from different instruments. For example, studies using IMAGE-FUV [3, 5], TIMED 
GUVI [3], TOPEX altimeter TEC [6], COSMIC occultation density [7, 8], CHAMP [9] and ROCSAT-1 [10] satellite in situ electron densities, and ground-based global total electron content (TEC) maps [1] are among those that have reported signature of global wave number-four. However, these longitudinal four-cell patterns cannot be explained by factors such as F-region wind, magnetic field strength, or the offset between magnetic and geographic equators. Instead, this longitudinal variation has been attributed to the solar thermal tides, excited by latent heat in the troposphere $[11,12]$, which are considered to modify the wind-driven E-region dynamo which in turn modifies the ionospheric $\mathbf{E} \times \mathbf{B}$ drifts [5]. The longitudinal variation in the heating from ozone and water vapor due to land-sea differences and topography and nonlinear interaction between the migrating tide and gravity waves [13] could be considered additional sources for eastward propagating nonmigrating tides with zonal wave number 3 (De3), which is considered to be the possible cause for the formation of these longitudinal fourpeaked structure $[5,12]$.

Even though modeling results [9] and indirect estimation from the CHAMP accelerometer measurements [14] show the existence of longitudinal four-peaked structure on the neutral wind velocities, no observational evidence indicating the existence of wave number four structures in the neutral wind has been presented so far. Since the E-region dynamo is driven by the neutral winds at E-region altitudes (see [15] and references therein), experimental observations of the neutral wind that demonstrates the formation of the four-peaked structures could strengthen the suggestion mentioned above, namely, that any tidal changes in the strength of the winds are responsible for the formation of longitudinal modulation of the E-region dynamo fields, which then causes the creation of the wave number-four structures often observed in the ionosphere.

In this paper, for the first time, we show clear evidence from TIMED TIDI observations that the longitudinal/local time modulation of the winds forms longitudinal fourpeaked structures that move to the east as altitude increases. At the same time both ground-based GPS TEC, C/NOFS in situ density, and CHAMP topside GPS TEC show similar longitudinal four-peaked structures. At the same time the neutral wind also forms four-peaked structures. However, the neutral wind peaks are out of phase by nearly $180^{\circ}$ with the peaks of the ionospheric density distributions. Therefore, these simultaneous analyses of neutral wind signatures and in situ and GPS TEC observations of the EIA offer intriguing opportunities for new studies of the equatorial ionosphere, especially in understanding the physics of the wave numberfour structure and its formation mechanisms.

\section{Instruments and Data Analysis}

2.1. TIDI Wind Measurement. The TIMED Doppler Interferometer (TIDI) is a wind-measuring instrument designed to investigate the dynamics of the Earth's mesosphere and lower thermosphere-ionosphere (MLTI) from an altitude of 70 to $120 \mathrm{~km}$. The TIMED satellite orbits at an altitude of $625 \mathrm{~km}$ and the orbital inclination is $74.1^{\circ}$; TIDI measures the horizontal vector wind field with an accuracy of $3 \mathrm{~m} / \mathrm{s}$ and a vertical resolution of $2 \mathrm{~km}$ [16]. Using the limb scanning techniques that scan various upper atmosphere air glow layers by observing emissions from OI at $557.7 \mathrm{~nm}$ and rotational lines in the $\mathrm{O}_{2}(0-0)$ atmospheric band at $762 \mathrm{~nm}$, TIDI determines the Doppler shift and hence measures the daytime and nighttime neutral wind velocities [17]. A detailed description of the TIDI instrument can be found elsewhere [16, 17].

Every 60 days the TIDI sampling track covers the 24 hours of local time. This is known as one yaw period (satellite orientation with respect to the orbital flight direction). Hence we analyzed both zonal and meridional winds based on individual yaw period. Such analysis is based on the assumption that the climatological events, such as the formation of four cell structures and the mean wind magnitude did not change significantly during each 60 -day period. Therefore, we averaged wind velocities for every 60 day period.

2.2. C/NOFS PLP Density. The successfully launched Communication/Navigation Outage Forecasting System (C/NOFS) satellite [18] with a variety of instruments onboard has brought a great opportunity to the scientific community to obtain a better understanding of equatorial ionospheric electrodynamics. It is flying in a low inclination $\left(13^{\circ}\right)$ elliptical $(400 \times 870 \mathrm{~km}$ altitude $)$ orbit with an orbital period of $100 \mathrm{~min}$. The dual disk probe PLP (Planar Langmuir Probe), which is designed to provide high time resolution measurements of in situ density irregularities by sensing ionospheric plasma density, is one of many instruments on board C/NOFS.

C/NOFS provides continuous measurements of the lowlatitude ionospheric ion density which is very important to study the climatological features of the equatorial ionosphere, like the formation of the four-cell structures.

2.3. Ground- and Space-Based GPS TEC. The GPS constellation currently consists of 29 satellites orbiting at $\sim 55^{\circ}$ inclination in six distinct orbital planes and at $\sim 20,200 \mathrm{~km}$ altitude $(\sim 4.2 \mathrm{~L})$. Each satellite broadcasts two L-band signals at frequencies $f_{1}=1.57542 \mathrm{GHz}$ and $f_{2}=1.2276 \mathrm{GHz}$. Owing to the dispersive nature of the ionosphere, dual frequency GPS measurements can provide the total electron content (TEC), by computing the differential phases of the code and carrier phase measurements recorded at ground based GPS receivers. Details of TEC calculation from GPS observations are described in several papers ([19] and references therein).

Similarly, LEO satellites that are equipped with dualband frequency GPS receivers, such as CHAMP $[20,21]$, offer the opportunity for remote sensing and monitoring of the topside ionosphere and plasmasphere regions. GPS receivers on board continuously receive dual frequency signals transmitted by GPS satellites. The integrated electron density along the ray path between the LEO GPS receiver and a GPS satellite can be derived in the same way as the groundbased GPS TEC [20]. 


\section{Observations}

The global longitudinal variability of the equatorial electron density, as a function of local time, can be monitored using the GPS TEC estimated from the data recorded by the fast growing number of GPS receivers around the world. Figure 1 shows a typical example of the 2D (latitude/longitude) variation of the global GPS TEC distribution. The maps represent two-month (July and August 2008) quiet days $(K p \leq 3)$ average of GPS TEC plotted for every twohour local time sectors. The local time coverage is given at the top of each panel, and the white areas in each panel indicate regions that do not have data coverage. As can be seen in the figure the longitudinal wave number four structure (indicated by the four pink circles) starts to develop in the morning (bottom panel) and becomes fully developed in the afternoon (second and third panel from top) before starting to fade out during the early evening sector as shown in the top panel. The density peak over Asia is stronger, and its development through time is not as visible as in the other three peaks, especially after local midday (third panel from bottom). The very interesting feature that can be noticed in Figure 1 is the density at the locations of those four peaks expanded to higher latitudes in the westward direction. The horizontal bold and faint dashed lines represent the geomagnetic equator and the equatorial anomaly peak region, respectively.

Figure 2 shows an example of the 60-day data averaged and binned into $5^{\circ}$ longitude $\times 2.5^{\circ}$ latitude bins. Each panel represents a different two-hour local time sector which is given at the top of each panel, and the panels cover the period from the morning (bottom panel) to late afternoon (top panel). Our analysis periods extend only from July to August 2008. In order to avoid the effects of storm-driven winds, only magnetically quiet periods $(K p \leq 3)$ were included. The positive and negative components represent eastward and westward wind directions, respectively. The left and right panels indicate the zonal wind estimated at an altitude of $105 \mathrm{~km}$ and $110 \mathrm{~km}$ altitudes, respectively. The zonal winds, as shown in both left and right panels, illustrate clear longitudinal structures with clear wave number four structures. The wave number four structures become more pronounced in the morning and early afternoon sectors as shown in the first three panels from the bottom. Even during late afternoon (the top two panels) the wave number four structures are visible but are not as pronounced as those during earlier local time sector. Since TIDI instrument measures the neutral wind from 80 to $103 \mathrm{~km}$ altitude during nighttime [17], it was not possible to see if the four-cell pattern persists in the zonal wind data after 1800 LT at $105 \mathrm{~km}$ and $110 \mathrm{~km}$ altitude. On the other hand, the meridional wind component does not show any clear structure of the wave number four signatures as is evident from Figure 3, which shows the meridional wind component in a similar fashion to Figure 2. The horizontal dashed curves, both in Figures 2 and 3, depict the location of the geomagnetic equator.

We also analyzed the ion in situ density data obtained from PLP measurements on board C/NOFS satellite. The three-month average ion density is binned into $5^{\circ}$ longitude and 30-minute local time bin. In order to get reasonable global data coverage for the criteria mentioned below, we averaged three-month data instead of two months. Therefore, for every three months, we produce one global (solar local time at the satellite position as function of longitude) ionospheric density structure, showing the climatological features of the low-latitude ionosphere such as the wave number-four structures. Figure 4 shows a typical example for the statistical 2D (local-time/longitude) global ionospheric in situ density of C/NOFS during August-October 2008. The top panel shows the in situ density averaged when the satellite was below $470 \mathrm{~km}$ altitude for the whole latitudes range $\left( \pm 13^{\circ} \mathrm{N}\right.$ geographic latitude), and for only $\pm 8^{\circ}$ magnetic latitudes coverage (second from top panel). In both panels the wave number four structures are evident, especially in the late afternoon sector centered at about 1700 local time. However, during early morning local time sector only three peaks are observed with the peak over Africa not visible at this time. The bottom two panels in Figure 4 show the zonal and meridional wind components observed by TIDI instrument onboard TIMED satellite. Here we averaged the winds from $-30^{\circ}$ to $30^{\circ}$ latitude. Since the winds are more symmetric along the geographic equator than geomagnetic equator, taking this latitude range is reasonable. The $2 \mathrm{D}$ (local time/longitude) maps of the zonal and meridional winds indicate that the zonal component is more structured than meridional component.

Similarly, the GPS receiver on board CHAMP satellite can also provide statistical images of the longitudinal variability of the topside ionospheric density distribution. The top panel in Figure 5 shows the three-month (August-October 2008) quiet days average of topside TEC distribution for 15001700 local time sectors. The latitude/longitude 2D variations of the topside GPS TEC indicate clear wave number four structures in the topside ionospheric density distribution. The bottom two panels present the latitude/longitude maps of the zonal (middle panel) and meridional (bottom panel) wind components averaged for the same time period as for the topside GPS TEC. Again, the zonal wind component shows more structured features, including the wave number four structure primarily in the southern hemisphere.

\section{Discussion and Conclusion}

The E-region tidal winds contribute to the east-west electric field everywhere in the equatorial and midlatitude ionosphere. During the daytime, horizontal wind motions in the E-region create currents that produce polarization electric fields in regions where there are conductivity gradients. These electric fields, which are eastward during the daytime at the equatorial region, in turn produce vertical uplift of the plasma around the magnetic equator. Immel et al. [5] first proposed that this was caused by the horizontal winds associated with the diurnal eastward propagating nonmigrating tides at the E-region altitudes, which is believed to be excited by the latent heat released in the tropical troposphere [11], modifying the $\mathbf{E} \times \mathbf{B}$ drifts associated with the E-region dynamo [22]. Such modification of 


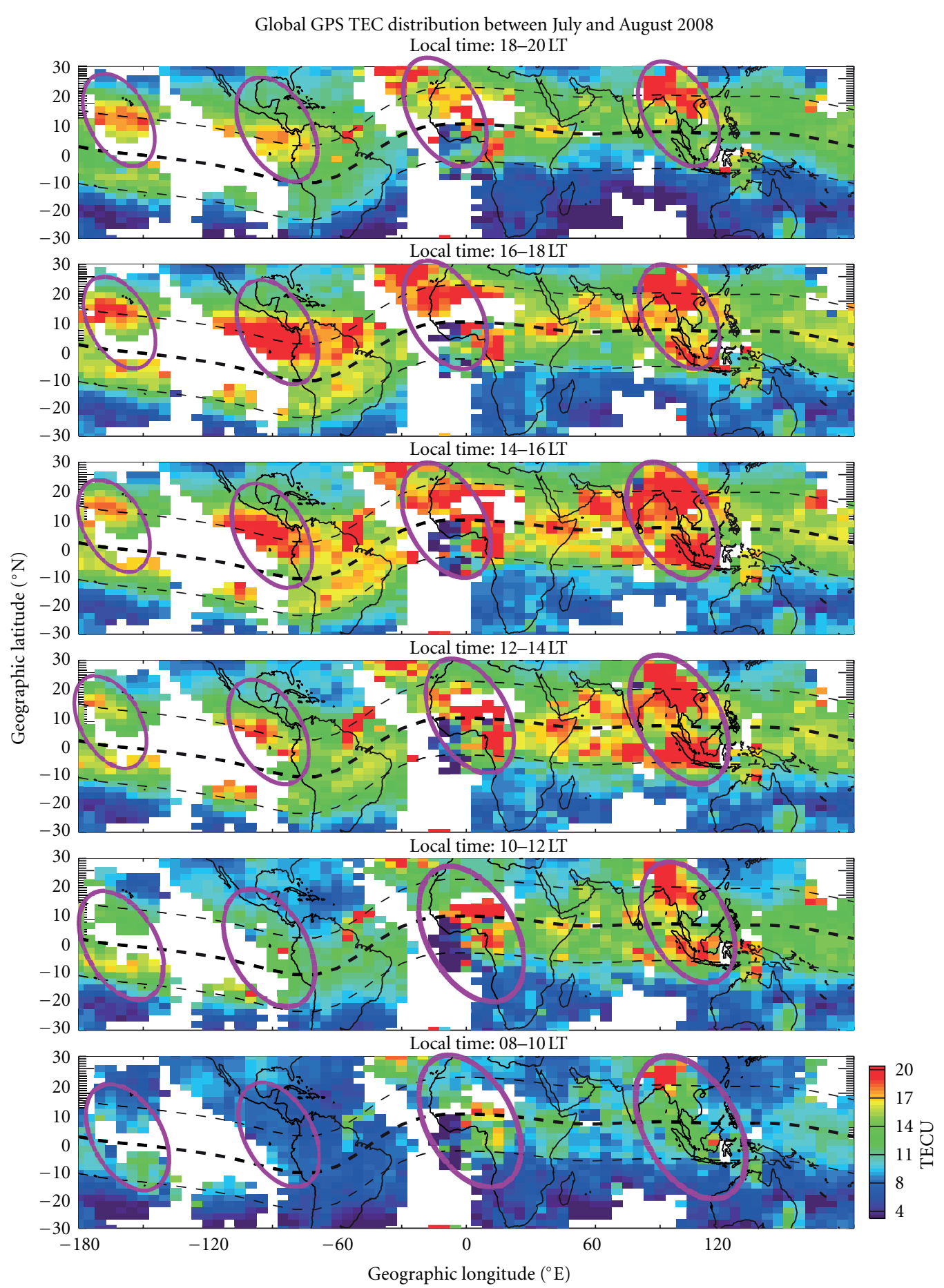

FIGURE 1: 2D (latitude/longitude) plot of the global two-month average GPS TEC distribution during magnetically quiet periods $(K p \leq 3)$ in July and August 2008. Each panel represents different local time sector shown at the top of each panel. The horizontal bold and faint dashed lines represent the geomagnetic equator and the equatorial anomaly peak region, respectively.

the global vertical drift velocities can cause the longitudinal dependence of ionospheric density distribution, such as the formation of the wave number four structures. Hagan et al. [12], using Thermosphere-Ionosphere-MesosphereElectrodynamics General Circulation Model (TIME-GCM) with lower boundary forcing from the Global Scale Wave
Model (GSWM), demonstrated that a wave number-four longitudinal variation for a fixed local time is due to the modulation of vertical equatorial plasma drifts in the Fregion through the dynamo action of the eastward propagating nonmigrating diurnal tide of wave number three (DE3). All these suggest that the longitudinal four-peaked 
TIMED TIDI zonal wind speed: July-August, 2008
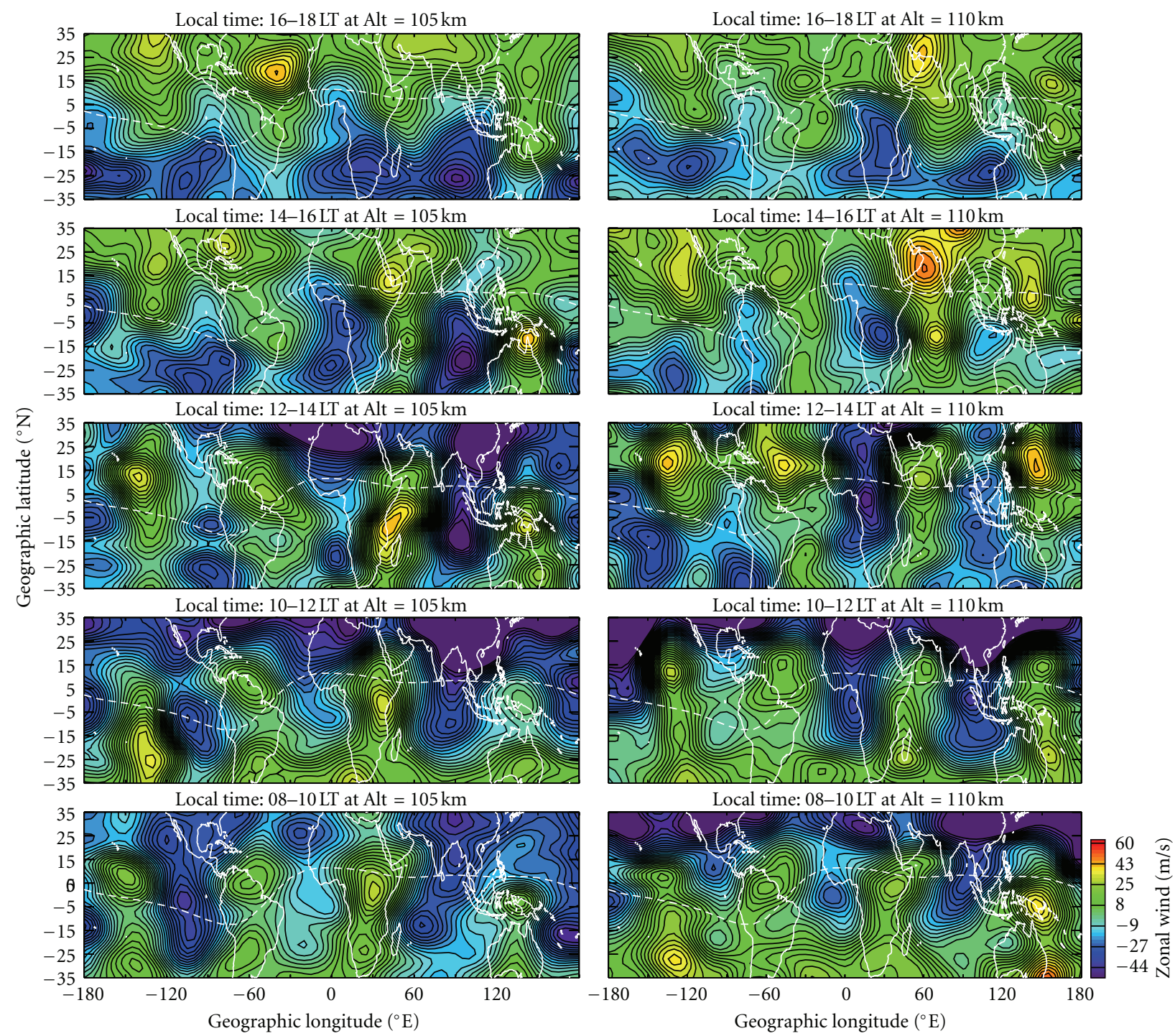

FIGURE 2: The 60 -day data average, into $5^{\circ}$ longitude $\times 2.5^{\circ}$ latitude bins, of the quiet periods $(K p \leq 3)$ zonal wind component observed by TIDI instrument onboard TIMED satellite at $105 \mathrm{~km}$ (left panels) and $110 \mathrm{~km}$ (right panels) altitudes in July and August 2008 . Each panel represents different two-hour local time sector which is given at the top of each panel. The positive and negative components represent eastward and westward wind direction, respectively.

structures are due to an internal driver rather from the lower thermosphere than external (magnetospheric or solar origin) influences. Therefore, to exclude the importance of such external drivers, for this study we only consider data that are recorded during magnetically quiet $(K p \leq 3)$ periods.

In the E-region at low latitudes the diurnal tide, which is a large-scale $24 \mathrm{~h}$ variation, dominates the neutral atmosphere motion, creating charge accumulation at dawn (positive charges) and dusk (negative charges) sectors (see [15]). The electric field resulting from these charge distributions is eastward during the day and westward at night. The thermal tides, excited by latent heat in the troposphere, also produce neutral wind in the E-region that possibly modifies the east-west electric field. In the E-region, where the Hall conductivity dominates, the neutral wind moves the ions in the direction of the wind, separating the charges, and probably causes the production of additional electric fields from polarization charges that accumulate wherever the wind-driven current has a divergence. Therefore, the electric field produced by these thermal tides may strengthen or weaken the net east-west electric fields (depending on the direction of the zonal wind) that are responsible for the ionospheric density distribution. The longitudinally structured zonal wind component (see Figures 2, 4, and 5) can produce eastward or westward electric field at different longitudes. If the thermal tidal zonal wind is directed to the west, it moves the ions westward and creates charge separation and thus causes the production of additional 
TIMED TIDI Meridional wind speed: July-August 2008
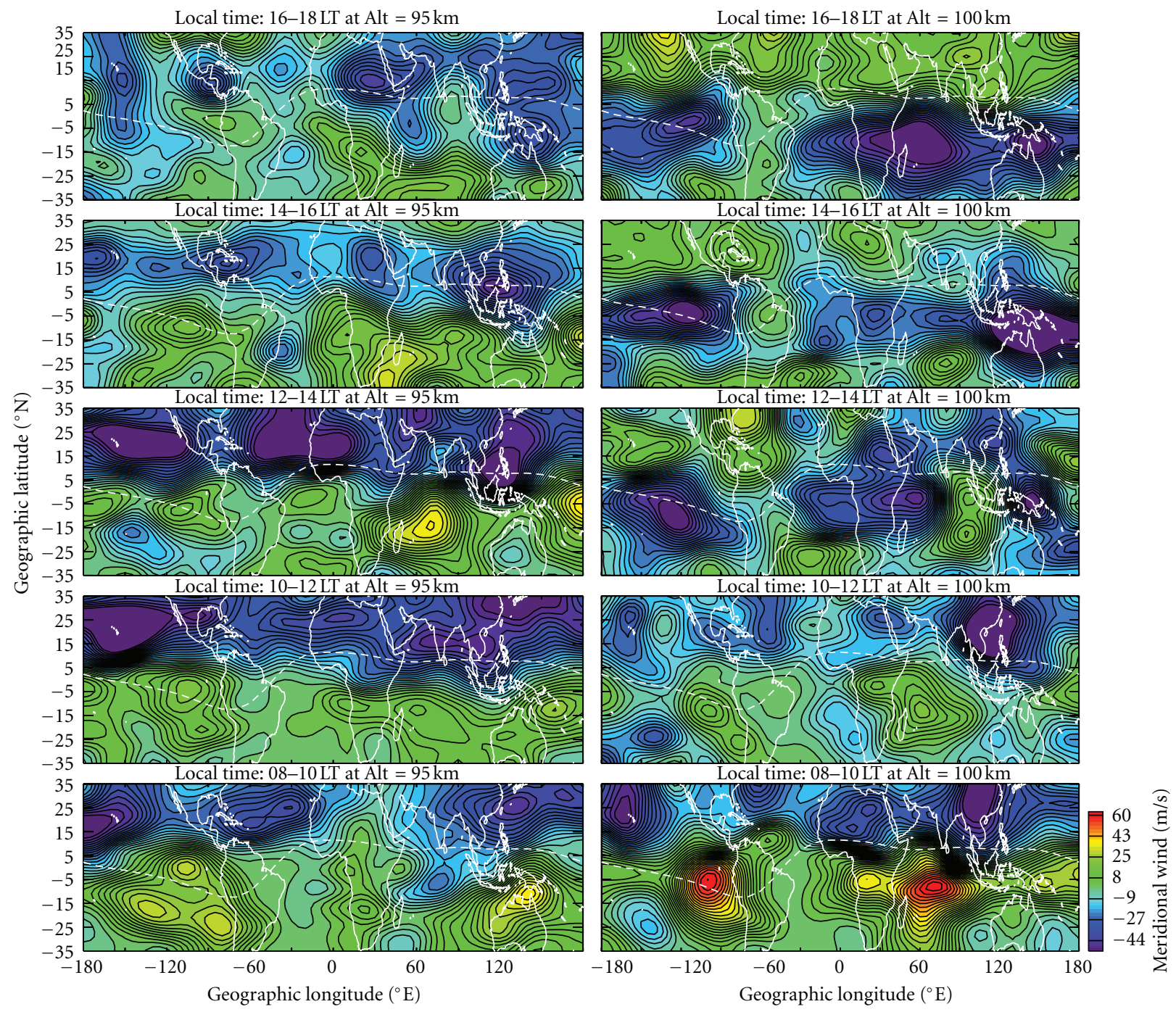

FIgURE 3: As for Figure 2 but it is for meridional wind component.

eastward electric field which adds to the net eastward electric field, and thus the vertical drift gets stronger. On the other, if the zonal wind is directed eastward, it produces additional westward electric field that weakens the net electric field and the vertical drift. This is clearly evident from our quiet days simultaneous observation of the global density and neutral wind distributions. The peaks of the wave number four structures are almost out of phase with the peaks of the neutral winds as shown in Figure 6. The figure shows the maximum density extracted from each longitudinal bins along the northern hemisphere anomaly peak region. The northern hemisphere is chosen due to its better data coverage compared to the southern hemisphere. Similar extraction technique was applied to the zonal wind data measured by the TIDI instrument onboard the TIMED satellite. Figure 6 shows the peaks of the global TEC distributions (blue curve) along with the peaks of the zonal winds at an altitude of $105 \mathrm{~km}$ (red curve) and $110 \mathrm{~km}$ (black curve), as a function of longitude during 1400-1600 (top panel) and 1600-1800 (bottom panel) local time. The vertical dashed lines indicate the longitudinal location of the peaks of the corresponding color curves. As can be seen in the figure, the peaks of the zonal winds shift eastward as the tidal wind propagates upward, and this is more clearly visible at the top panel. This is consistent with the eastward propagating nonmigrating tides with wave number 3 (DE3), which is believed to be responsible for the modification of the longitudinal dependence of eastward electric field and thus vertical drift that alters the longitudinal distribution of low-latitude ionospheric density. The peaks of the zonal winds are also almost out of phase by 180 degree with the peaks of the global TEC. The neutral wind directions are almost westward at the longitudes where the peaks of ionospheric four-cell pattern are located; even if it is eastward, its magnitude is very weak. This is consistent with the physics description mentioned above. This is because of 
C/NOFS density August-October 2008

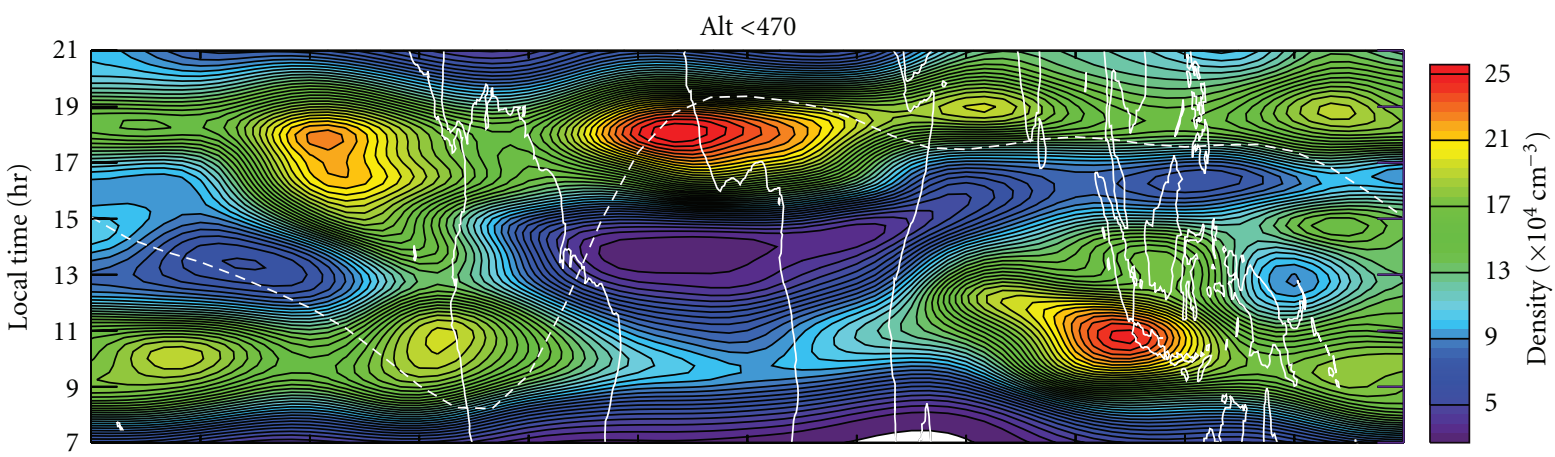

Mlat: $-8.0-+8^{\circ} \mathrm{N} ;$ Alt $<470$

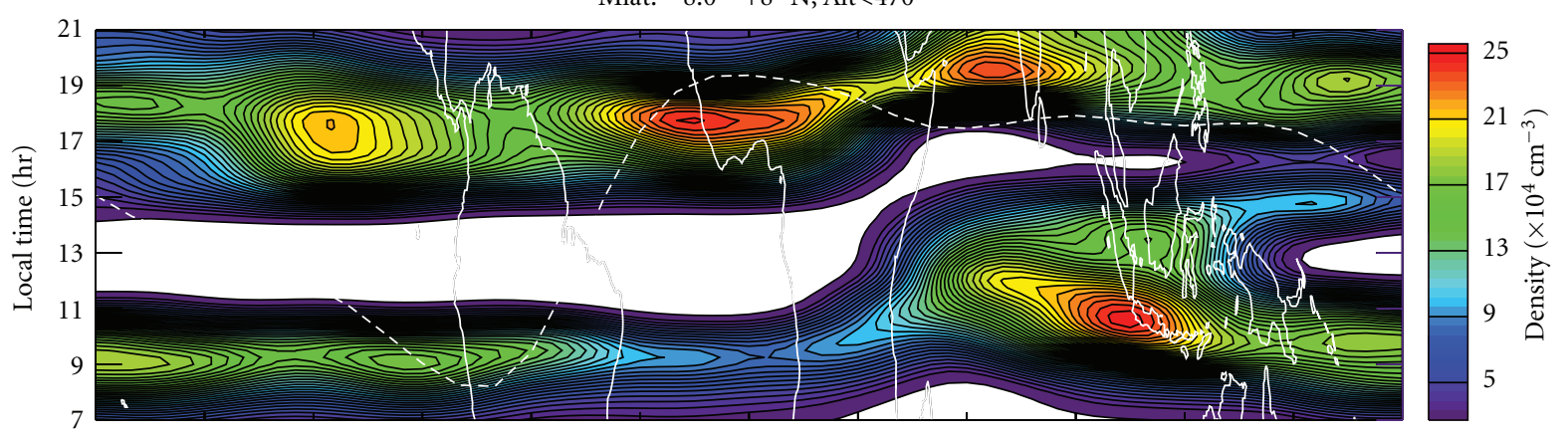

TIMED TIDI zonal wind: August-October 2008

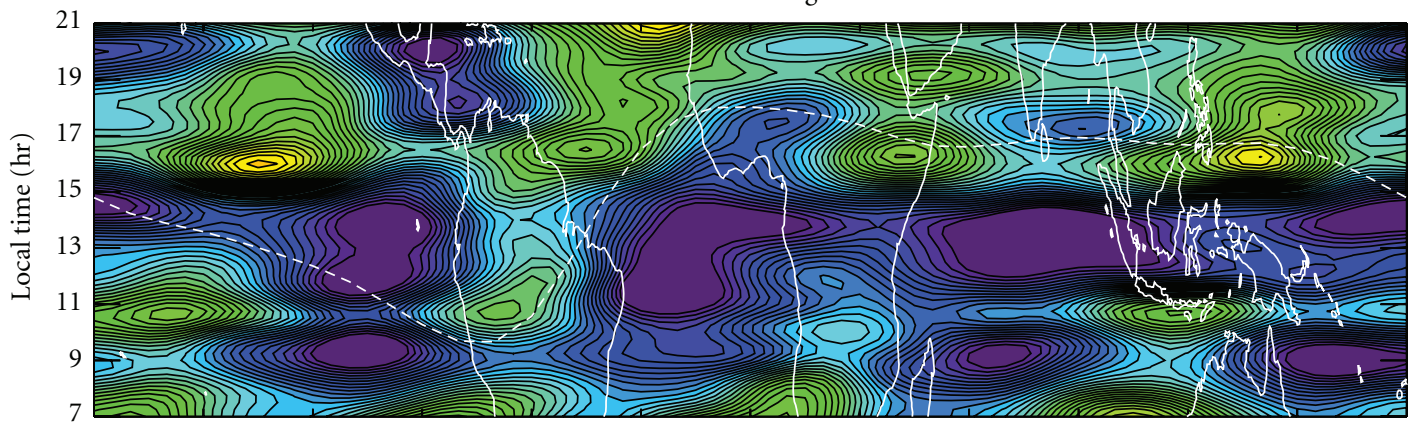

$100 \mathrm{~km}$

TIMED TIDI meridional wind: August-October 2008

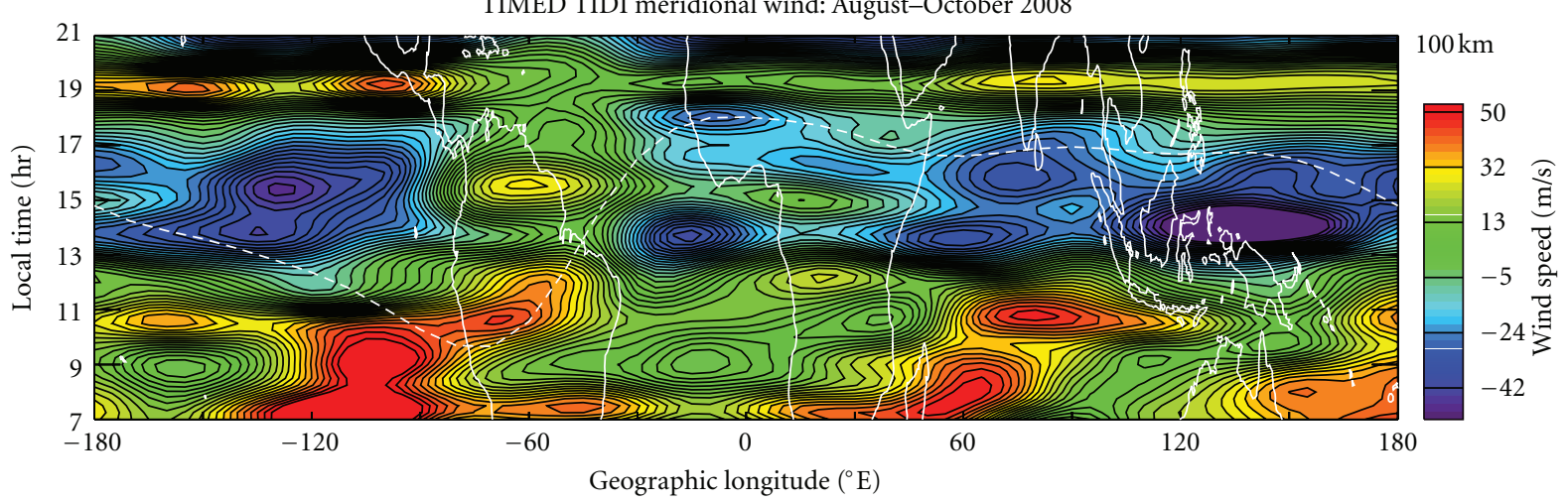

FIgURE 4: Plot of the statistical 2D (local-time/longitude) ionospheric in situ density observed during quiet days (Kp $\leq 3$ ) by PLP instrument onboard C/NOFS during August-October 2008. The top panel shows the in situ density average when the satellite was below $470 \mathrm{~km}$ altitude for the whole latitudes $\left( \pm 13^{\circ} \mathrm{N}\right.$ geographic latitude), and for only $\pm 8^{\circ}$ magnetic latitudes coverage (second from top panel). The bottom two panels show the zonal and meridional wind components observed by TIDI instrument onboard TIMED satellite. 
CHAMP average TEC map during 244-304, 2008 at 15-17 LT

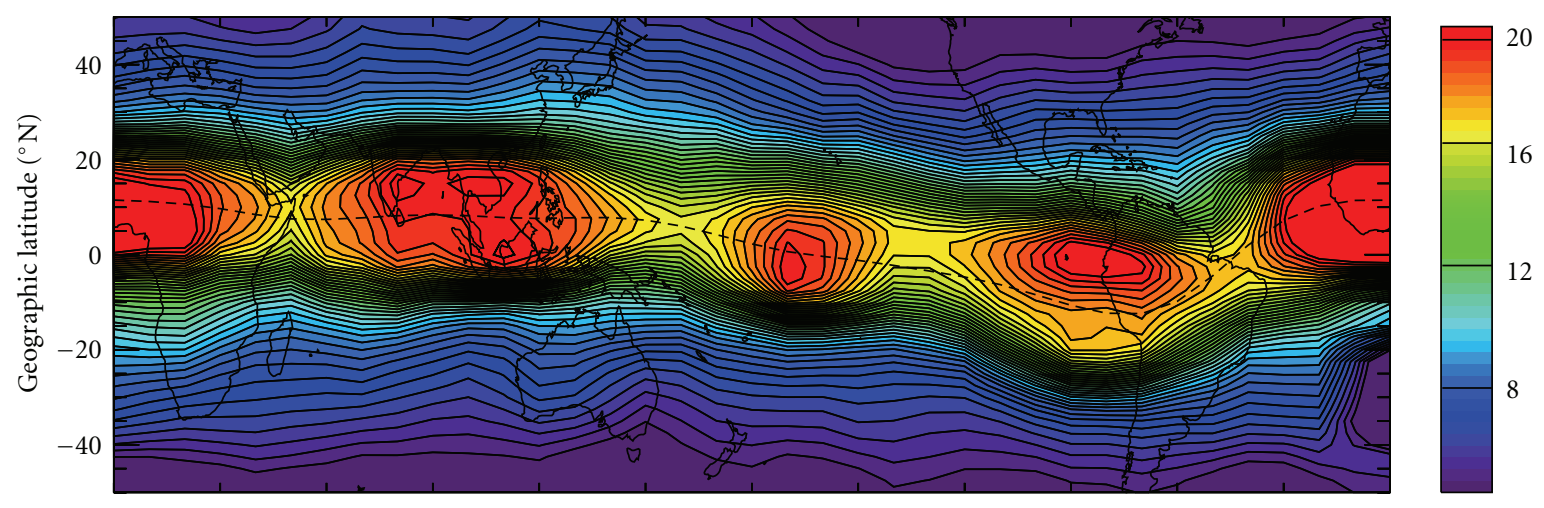

TIMED TIDI zonal wind: 244-304, 2008 at 15-17 LT

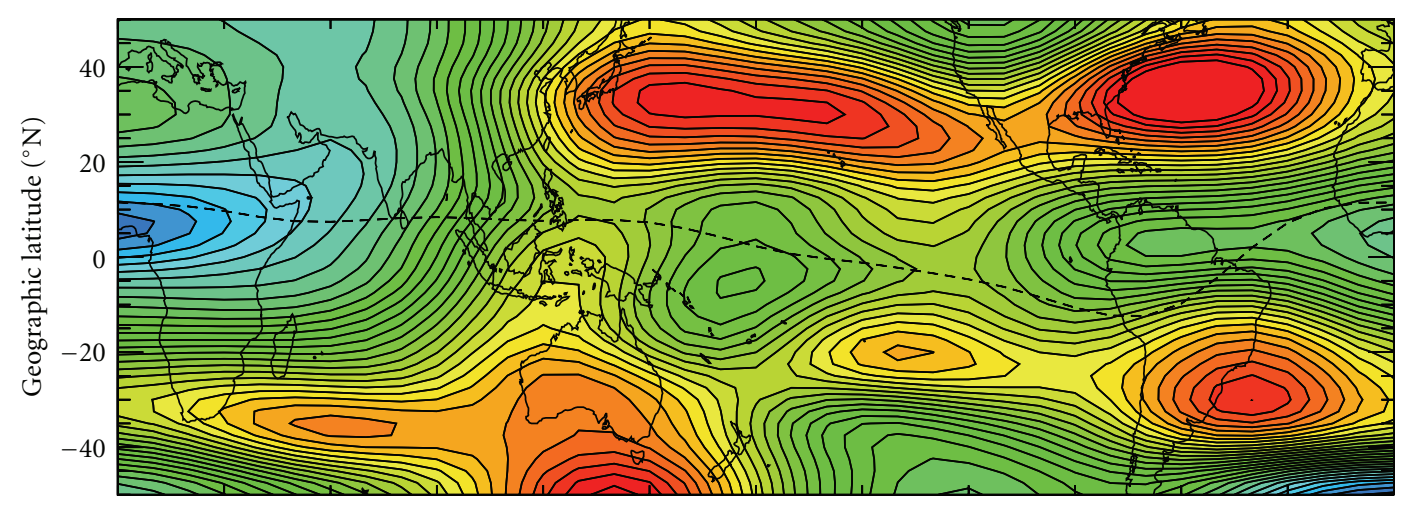

TIMED TIDI meridional wind: $244-304,2008$ at $15-17$ LT

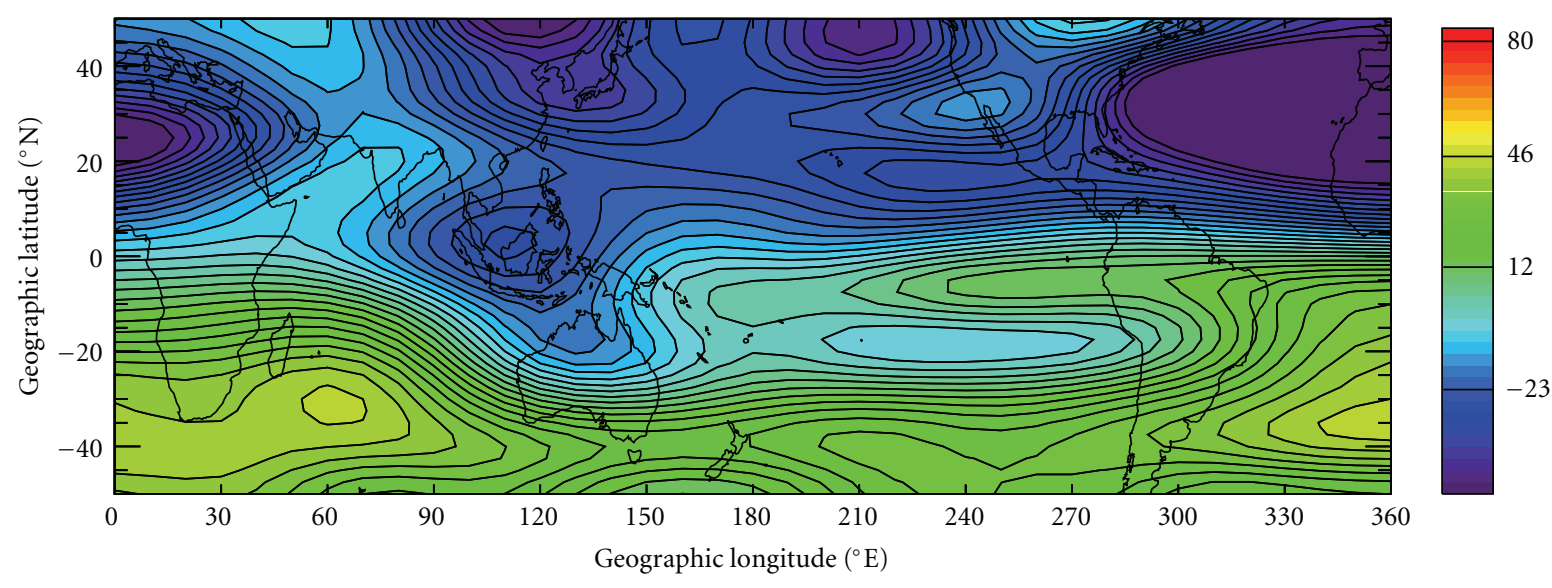

FIGURE 5: The top panel shows the quiet days $(K p \leq 3)$ three-month average of topside TEC distribution during August-October 2008 for 1500-1700 local time sectors. The bottom two panels present the latitude/longitude maps of the zonal (middle panel) and meridional (bottom panel) wind components averaged for same time period as for topside GPS TEC.

the electric field, resulting from these charge distributions by the winds in the E-region, maps along the magnetic field to the F-region where it produces vertical drift and redistributes the ionospheric plasma. At the same time, the meridional component of the tidal neutral wind (see Figure 3 ) does not show any clear longitudinal structure, indicating that the zonal component has a more significant effect than the meridional component on the modulation of the equatorial vertical drifts and thus the longitudinal dependence of the global density distribution. This could be due to the Kelvin wave mode of the DE3 tides that modulate the neutral winds [3]. The Kelvin wave, which is the first symmetric mode of the DE3, has strong zonal winds and only very weak meridional winds. Similarly, using measurements from 


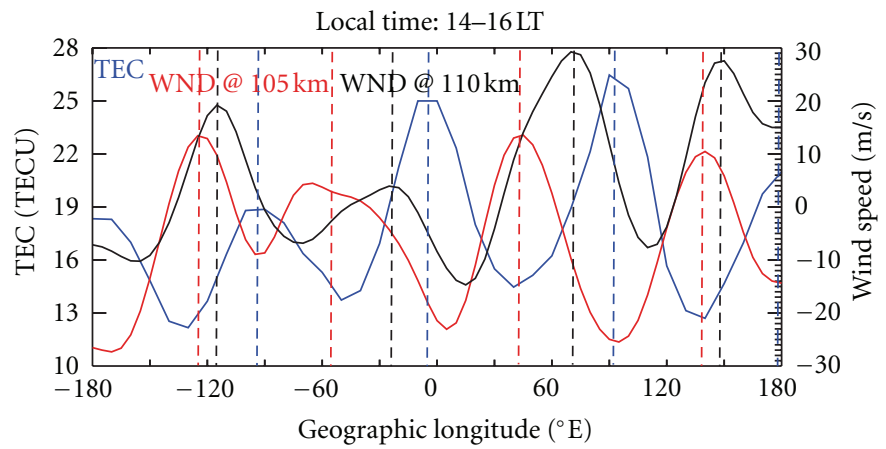

(a)

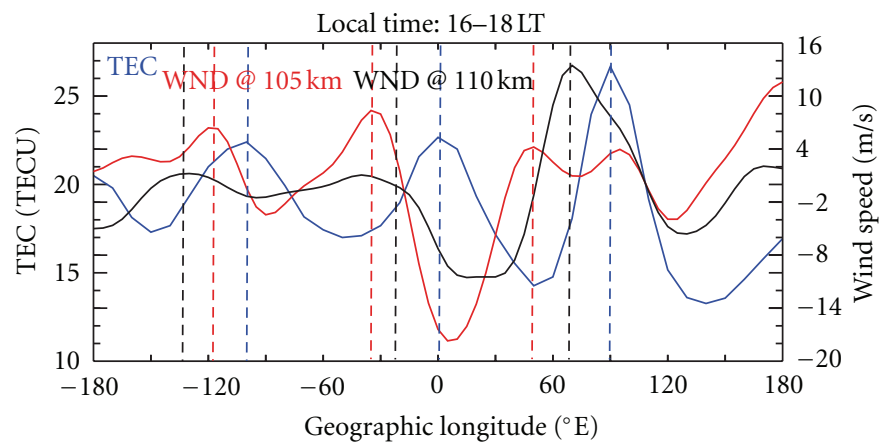

(b)

FIgURE 6: The peaks of the global TEC distributions (blue curve) along with the peaks of the zonal winds, measured by TIDI instrument onboard TIMED satellite at an altitude of $105 \mathrm{~km}$ (red curve) and $110 \mathrm{~km}$ (black curve), as a function of longitude during $1400-1600$ (top panel) and 1600-1800 (bottom panel) local time.

the CHAMP accelerometer, Lühr et al. [14] have quantified the zonal wind perturbations, associated with nonmigrating tides at F-region altitudes. They showed strong wave number four structure in the indirectly estimated zonal wind from CHAMP accelerometer observations. Lühr et al. [14] also found that the peaks of the four-cell pattern of zonal wind are out of phase by 180 degree with the density peaks, which is consistent with our result.

The other very important feature we observed from our global TEC distribution is the westward expansion of the TEC peaks, indicated by the pink circles in Figure 1. The global TEC four-cell pattern, which is stronger in the summer northern hemisphere, becomes visible around early morning local time (see bottom panel), especially at about the $100^{\circ} \mathrm{E}$ where the TEC formed a clear anomaly structure. The transequatorial wind could be responsible for the formation of asymmetry anomaly peaks, stronger in the northern hemisphere [15]. The four-cell pattern then persists until midnight with more pronounced four peaks and clear westward expansion between 1400 and 1800 local time sector as shown in the second and third panels from the top in Figure 1. On the other hand, except in a few cases shown in Figure 2, the zonal wind peaks do not show a clear westward expansion. Even for the local time/longitude plot (see Figure 4) the zonal wind forms a clear eastward expansion pattern with time. As mentioned above, the global TEC distribution presented in Figure 1 represents only the quiet day $(K p \leq 3)$ average, and thus there is no reason to claim that the westward expansion of the TEC peaks is due to subauroral polarization stream (SAPS) $[23,24]$, which are of magnetospheric origin or expanded high-latitude convection [25] electric fields as mechanisms for producing such westward expansion of enhanced TEC. Instead, most possibly it may be associated with the driver from the lower thermosphere, but further modeling investigation is required to understand the physics behind such westward expansion of the TEC enhancements at peaks of the four-cell pattern.

In conclusion, the eastward propagating nonmigrating tide with wavenumber-3 (DE3) excited by latent heat release in the tropical troposphere is believed to be responsible for the modification of the tidal winds which in turn are responsible for the modulation of the E-region dynamo and thus for the longitudinal variability of ionospheric density distribution. For the first time, we present simultaneous observations of the global wind structures in the E-region and the ionospheric density distributions. Our results show that the longitudinally structured tidal zonal wind component could be responsible for the formation of wave number four pattern of the equatorial anomaly. A good correlation between the tidally modulated zonal winds and the equatorial anomaly indicates that the zonal winds may provide the most likely mechanism responsible for the longitudinal dependence of the global density distribution, including the formation of the wave number four structures. 
This may occur through the longitudinal variation in the thermal zonal winds which drive the dynamo effect, such as the westward (eastward) tidally modified zonal wind which can produce additional eastward (westward) electric field that can strengthen (weaken) the net electric field and thus the vertical drift velocity at specified longitudinal sectors. We also noticed the westward expansion of the enhanced TEC at longitudes where the four-cell pattern peaks are located. However, it is not clear what causes such westward expansion during quiet periods $(K p \leq 3)$ which allows us to rule out the effectiveness of both SAPS or expanded convection electric field as producing such westward expansion of enhanced ionospheric density. The results presented here highlight the importance of understanding the physics behind such expansion, and more modeling investigation is required to develop a better understanding of the feature.

\section{Acknowledgments}

This work was supported by NASA LWS and Geospace Science programs (NNX11AP02G and NNX09AR84G) and AFOSR YIP Grant (FA9550-10-1-0096). The authors are indebted to the Haystack MIT for the global GPS TEC, C/ NOFS team for the in situ density, CHAMP team for the GPS TEC data, and TIDI-TIMED team for the neutral wind data.

\section{References}

[1] W. B. Wang, J. Lei, A. G. Burns et al., "Ionospheric electric field variations during a geomagnetic storm simulated by a coupled magnetosphere ionosphere thermosphere (CMIT) model," Geophysical Research Letters, vol. 35, no. 18, Article ID L18105, 2008.

[2] E. Yizengaw, M. B. Moldwin, Y. Sahai, and R. De Jesus, "Strong postmidnight equatorial ionospheric anomaly observations during magnetically quiet periods," Journal of Geophysical Research A, vol. 114, no. 12, Article ID A12308, 2009.

[3] S. L. England, S. Maus, T. J. Immel, and S. B. Mende, "Longitudinal variation of the E-region electric fields caused by atmospheric tides," Geophysical Research Letters, vol. 33, no. 21, Article ID L21105, 2006.

[4] E. Sagawa, T. J. Immel, H. U. Frey, and S. B. Mende, "Longitudinal structure of the equatorial anomaly in the nighttime ionosphere observed by IMAGE/FUV," Journal of Geophysical Research A, vol. 110, no. 11, Article ID A11302, 2005.

[5] T. J. Immel, E. Sagawa, S. L. England et al., "Control of equatorial ionospheric morphology by atmospheric tides," Geophysical Research Letters, vol. 33, no. 15, Article ID L15108, 2006.

[6] L. Scherliess, D. C. Thompson, and R. W. Schunk, "Longitudinal variability of low-latitude total electron content: tidal influences," Journal of Geophysical Research A, vol. 113, no. 1, Article ID A01311, 2008.

[7] C. H. Lin, C. C. Hsiao, J. Y. Liu, and C. H. Liu, "Longitudinal structure of the equatorial ionosphere: time evolution of the four-peaked EIA structure," Journal of Geophysical Research A, vol. 112, no. 12, p. A12305, 2007.

[8] G. Liu, T. J. Immel, S. L. England, K. K. Kumar, and G. Ramkumar, "Temporal modulations of the longitudinal structure in F2 peak height in the equatorial ionosphere as observed by
COSMIC," Journal of Geophysical Research A, vol. 115, no. 4, Article ID A04303, 2010.

[9] S. L. England, T. J. Immel, E. Sagawa et al., "Effect of atmospheric tides on the morphology of the quiet time, postsunset equatorial ionospheric anomaly," Journal of Geophysical Research A, vol. 111, no. 10, Article ID A10S19, 2006.

[10] H. Kil, E. R. Talaat, S. J. Oh, L. J. Paxton, S. L. England, and S. Y. Su, "Wave structures of the plasma density and vertical e $\times$ B drift in low-latitude F region," Journal of Geophysical Research A, vol. 113, no. 9, Article ID A09312, 2008.

[11] M. E. Hagan and J. M. Forbes, "Migrating and nonmigrating diurnal tides in the middle and upper atmosphere excited by tropospheric latent heat release," Journal of Geophysical Research D, vol. 107, no. 24, pp. 21-22, 2002.

[12] M. E. Hagan, A. Maute, R. G. Roble, A. D. Richmond, T. J. Immel, and S. L. England, "Connections between deep tropical clouds and the Earth's ionosphere," Geophysical Research Letters, vol. 34, no. 20, Article ID L20109, 2007.

[13] C. McLandress and W. E. Ward, "Tidal/gravity wave interactions and their influence on the large- scale dynamics of the middle atmosphere: model results," Journal of Geophysical Research, vol. 99, no. 4, pp. 8139-8155, 1994.

[14] H. Lühr, K. Häusler, and C. Stolle, "Longitudinal variation of F region electron density and thermospheric zonal wind caused by atmospheric tides," Geophysical Research Letters, vol. 34, no. 16, Article ID L16102, 2007.

[15] R. A. Heelis, "Electrodynamics in the low and middle latitude ionosphere: a tutorial," Journal of Atmospheric and SolarTerrestrial Physics, vol. 66, no. 10, pp. 825-838, 2004.

[16] T. L. Killeen, Q. Wu, S. C. Solomon et al., "TIMED Doppler Interferometer: overview and recent results," Journal of Geophysical Research A, vol. 111, no. 10, Article ID A10S01, 2006.

[17] J. Oberheide, Q. Wu, T. L. Killeen, M. E. Hagan, and R. G. Roble, "Diurnal nonmigrating tides from TIMED Doppler Interferometer wind data: monthly climatologies and seasonal variations," Journal of Geophysical Research A, vol. 111, no. 10, Article ID A10S03, 2006.

[18] O. de La Beaujardière, L. Jeong, B. Basu et al., "C/NOFS: a mission to forecast scintillations," Journal of Atmospheric and Solar-Terrestrial Physics, vol. 66, no. 17, pp. 1573-1591, 2004.

[19] E. Yizengaw, M. B. Moldwin, P. L. Dyson, and T. J. Immel, "Southern Hemisphere ionosphere and plasmasphere response to the interplanetary shock event of 29-31 October 2003," Journal of Geophysical Research A, vol. 110, no. 9, Article ID A09S30, 2005.

[20] A. J. Mannucci, B. T. Tsurutani, B. A. Iijima et al., "Dayside global ionospheric response to the major interplanetary events of October 29-30, 2003 "Halloween Storms"," Geophysical Research Letters, vol. 32, no. 12, pp. 1-4, 2005.

[21] E. Yizengaw, M. B. Moldwin, A. Komjathy, and A. J. Mannucci, "Unusual topside ionospheric density response to the November 2003 superstorm," Journal of Geophysical Research A, vol. 111, no. 2, Article ID A02308, 2006.

[22] W. A. Hartman and R. A. Heelis, "Longitudinal variations in the equatorial vertical drift in the topside ionosphere," Journal of Geophysical Research A, vol. 112, no. 3, Article ID A03305, 2007.

[23] J. C. Foster, P. J. Erickson, A. J. Coster, J. Goldstein, and F. J. Rich, "Ionospheric signatures of plasmaspheric tails," Geophysical Research Letters, vol. 29, no. 13, article 1623, 4 pages, 2002.

[24] E. Yizengaw, J. Dewar, J. MacNeil et al., "The occurrence of ionospheric signatures of plasmaspheric plumes over different 
longitudinal sectors," Journal of Geophysical Research A, vol. 113, no. 8, Article ID A08318, 2008.

[25] R. A. Heelis, J. J. Sojka, M. David, and R. W. Schunk, "Storm time density enhancements in the middle-latitude dayside ionosphere," Journal of Geophysical Research A, vol. 114, no. 3, Article ID A03315, 2009. 

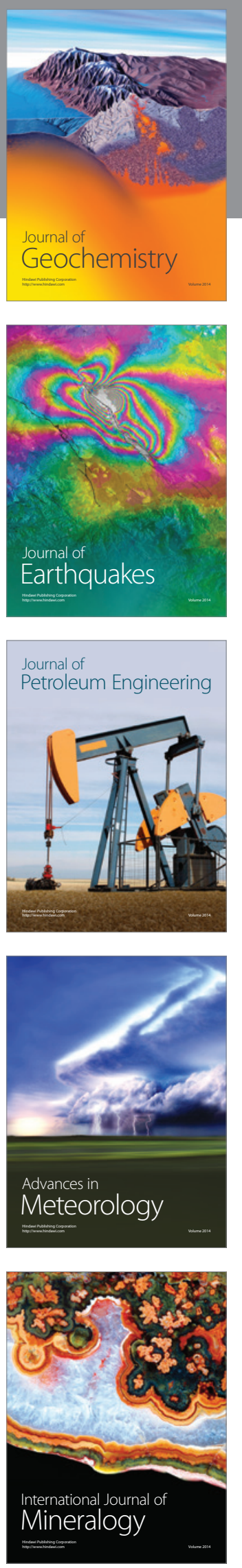
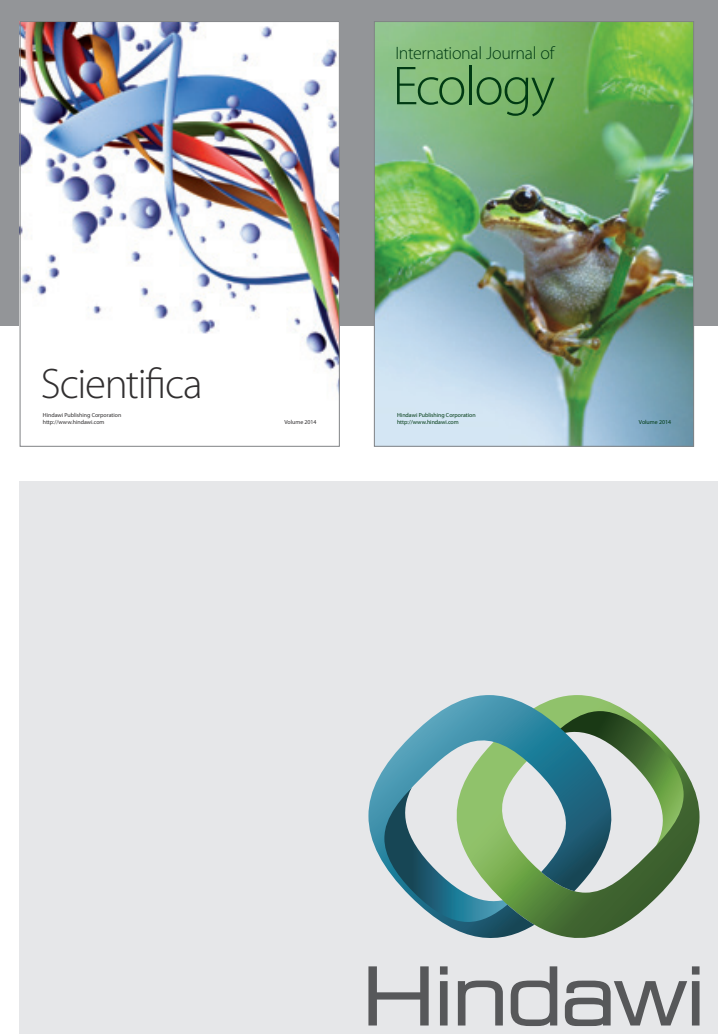

Submit your manuscripts at http://www.hindawi.com
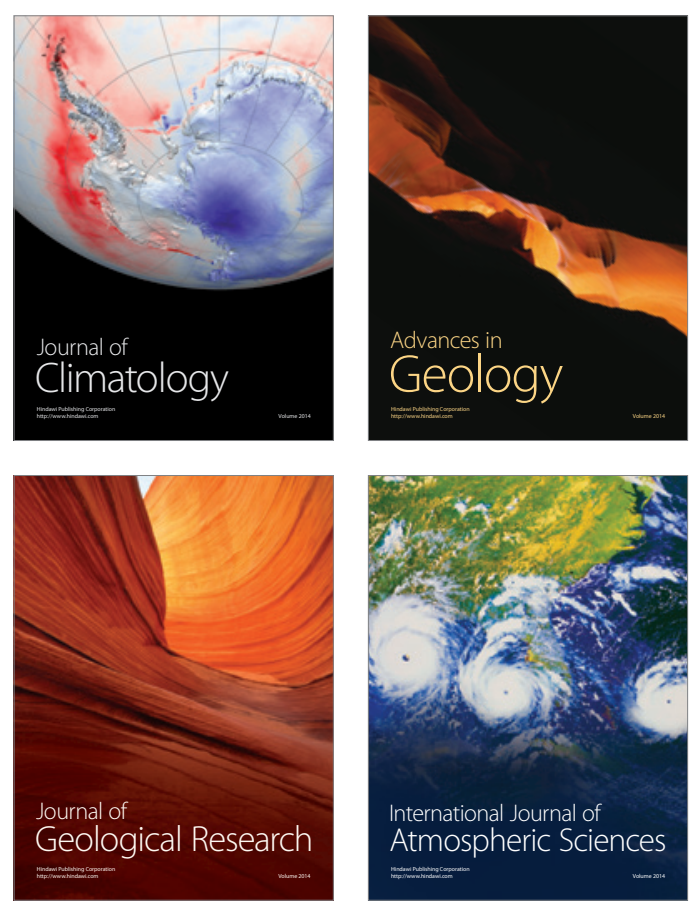
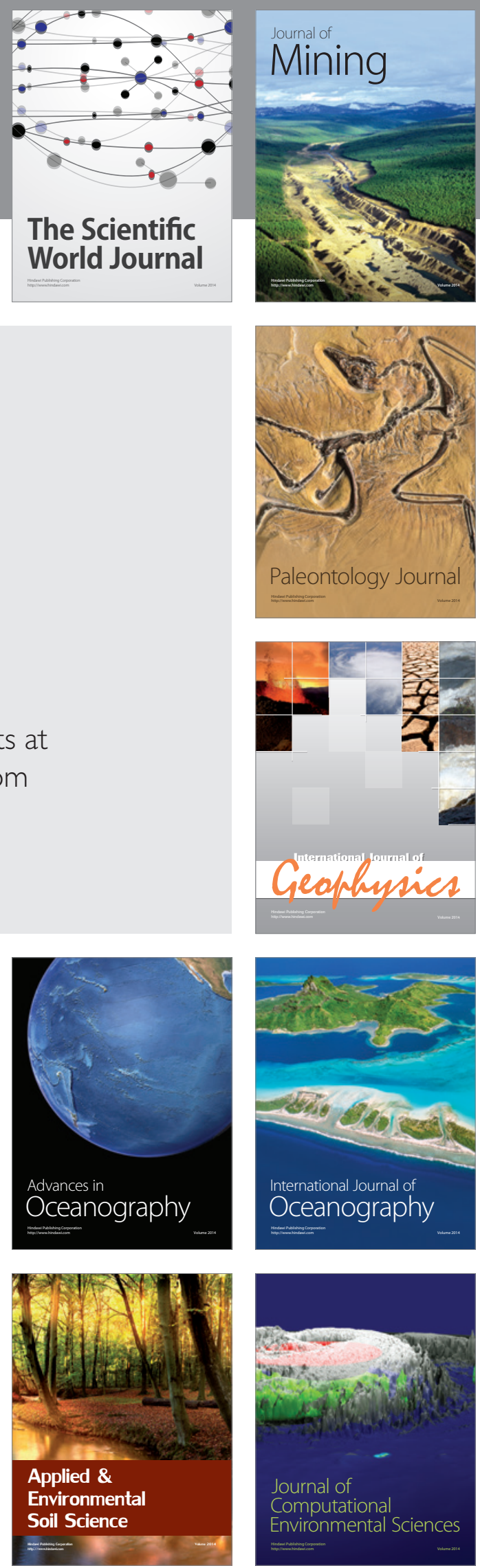